\title{
Das Ende der Sicherungsverwahrung
}

Bernd-Rüdeger Sonnen

Kein Gericht, also auch nicht das Urteil des Bundesverfassungsgerichtes vom 4.5.2011, kann die gesamte Gesetzgebung und Praxis der Sicherungsverwahrung seit den Erweiterungen und Verschärfungen 1998 bis zur Neuregelung 2011 behandeln. Aber dennoch wird diese Entscheidung dazu führen, dass sich Bund und Länder bis Ende 2013 zu einem freiheitsorientierten und auf Therapie gerichteten Gesamtkonzept durchringen müssen. Anlass für eine völlige Neuorientierung ist die Forderung, dass zwischen Strafverbüßung und Sicherungsverwahrung ein angemessener Abstand eingehalten werden muss. Bedenkt man, dass das Strafvollzugsgesetz bereits viele der Forderungen enthält, die das Bundesverfassungsgericht für die Verwahrung höchst gefährlicher und loder gestörter Täter fordert, kann man gespannt sein, wie die Bundes- und Landesgesetzgebung dies umsetzen wird. Im folgenden fasst Bernd-Ruedeger Sonnen kurz die Ergebnisse dieses Urteils zusammen und berichtet von der mündlichen Verhandlung. Christine Morgenstern wird dann auf S. $55 \mathrm{ff}$ sich kritisch mit dem vom Gericht noch nicht präzisierten Begriff der psychischen Störung auseinander setzen, welche nach dem Therapieunterbringungsgesetz (ThUG) eine Unterbringung rechtfertigt. Eigentlich sollte sich die Bundesgesetzgebung überlegen, ob der viel zu enge Anwendungsbereich des ThUG nach dieser Klarstellung des BVerfG nicht erweitert werden sollte, um eine eng definierte Therapieunterbringung ganz grundsätzlich an die Stelle der 1934 geschaffenen und mit rechtsstaatlichen Grundsätzen unvereinbaren Sicherungsverwahrung zu setzen.

\section{Die Entscheidung vom 4.5.2011}

In dem sorgfältig begründeten, im sensiblen Umgang mit den Entscheidungen des EGMR sowie den Sicherheitsinteressen der Allgemeinheit durchaus beeindruckenden Urteil vom 4.5.2011 wird festgestellt, dass die Vorschriften über die rückwirkend angeordnete oder verlängerte Freiheitsentziehung in der Sicherungsverwahrung ( $\mathbb{S} 66$ b Abs. 2, 67d Abs. 3 Satz 1 StGB, 7 Abs. 2 JGG) mit Art. 2 Abs. 2 Satz 2 i.v.m. Art. 104 Abs. 1 Satz 1 (Freiheitsentziehung) und i.v.m Art. 20 Abs. 3 GG (rechtsstaatliches Vertrauensschutzgebot, Fehlen eines dem verfassungsrechtlichen Abstandsgebot entsprechenden Gesamtkonzept der Sicherungsverwahrung) unvereinbar und die angefochtene Entscheidungen( mit Ausnahme der vorläufigen Unterbringung) deswegen aufzuheben und die Rechtssachen zur erneuten Entscheidung zurückzuverweisen sind. Die verfassungswidrigen Vorschriften der Sicherungsverwahrung sind allerdings nicht für nichtig erklärt worden, sondern gelten bis zur gesetzlichen Neuregelung des geforderten Gesamtkonzeptes, längstens bis zum 31.3.2013 nach Maßgabe des verfassungsrechtlichen Urteils weiter: „Eine rückwirkend angeordnete oder verlängerte Freiheitsentziehung durch Sicherungsverwahrung kann daher nur noch als verhältnismäßig angesehen werden, wenn

- Der gebotene Abstand zur Strafe gewahrt wird,

- Eine hochgradige Gefahr schwerster Gewalt- oder Sexualstraftaten

- Aus konkreten Umständen in der Person oder dem Verhalten des Untergebrachten abzuleiten ist (vgl. bereits BGH NJW 2011,240,243) und
- Die Voraussetzungen des Art. 5 Abs. 1 Satz 2 Buchstabe e EMRK in der hier zugrundegelegten Auslegung erfüllt sind.

- Lediglich in solchen Ausnahmefällen kann noch von einem Überwiegen der öffentlichen Sicherheitsinteressen ausgegangen werden“ (BVerf, Urt.v. 4.5.2011, Rn 156).

Gerade im Hinblick auf den letztgenannten Aspekt bedarf es einer kompetenten Aufklärung der Bevölkerung und ihrer Sensibilisierung gegenüber Freiheits-, Grund- und Menschenrechten.

\section{Eindrücke aus der mündlichen Verhandlung vom 8.2.2011}

Gegenstand der mündlichen Verhandlung in Sachen Sicherungsverwahrung vor dem Zweiten Senat des Bundesverfassungsgerichts waren insgesamt fünf Verfassungsbeschwerden, die sich gegen die Fortdauer der Sicherungsverwahrung über die früher geltende Höchstgrenze von 10 Jahren hinaus bzw. gegen die nachträgliche Anordnung richten. Dazu gehört der bisher einzige Fall der nachträglichen Sicherungsverwahrung nach Jugendstrafrecht. Der Beschwerdeführer hatte sowohl gegen die Anordnung der einstweiligen Unterbringung in der Sicherungsverwahrung Beschwerde als auch gegen die gemäß $\$ 7$ Abs. 2 JGG nachträglich durch das LG Regensburg angeordnete Sicherungsverwahrung Revision eingelegt (jeweils erfolglos).

In der klar und sehr gut strukturierten Verhandlung ging es um die Verfassungsmäßigkeit der einschlägigen Bestimmungen des StGB und JGG, die unter drei verfassungsrechtlichen Aspekten behandelt und nun in der am 4.5.2011 verkündeten Entscheidung geklärt worden sind (Vereinbarkeit mit Art. 103 Abs. 2 - Gesetzlichkeitsprinzip -, Art. 2 Abs. 2 iVm Art. 20 Abs. 3 - Vertrauensschutzgebot - und Art. 103 Abs. 3 GG - Doppelbestrafungsverbot). Für den besonders umfangreichen zweiten Teil waren als Stichworte genannt: Qualifikation als echte/unechte Rückwirkung, Verhältnismäßigkeitsprüfung, Berücksichtigung der Wertungen von Art. 5 Abs. 1 und Art. 7Abs. 1 EMRK, Beachtung und gesetzliche Ausgestaltung des sog. Abstandsgebots, Schutzpflichten des Staates, Möglichkeiten und Grenzen der verfassungskonformen Auslegung sowie alternative Lösungswege (Handlungsmöglichkeiten nach dem Therapieunterbringungsgesetz - ThUG - und den Landesunterbringungsgesetzen, Führungsaufsicht, Bewährungshilfe und ambulante Maßnahmen).

Die ersten Stellungnahmen erfolgten durch die Bevollmächtigten der Beschwerdeführer, zunächst durch Jörg Kinzig und Gunter Widmaier. Kinzig (vgl. NK 4/2010 und JZ 2010, 689) kritisierte das Urteil BVerfGE 109, 133 v. 5.2.2004 und die neuen Strafvollzugsgesetze Bayerns und Niedersachsens, in denen eine Reform des Vollzuges der Sicherungsverwahrung nicht erfolgt ist. Widmaier betonte u.a. die „eklatante“ Verfassungs- und Menschenrechtswidrigkeit des neuen Art. 316 e EGStGB, der als Übergangsvorschrift zum Gesetz zur Neuordnung des Rechts der Sicherungsverwahrung und begleitenden Regelungen bestimmt, dass die Reform nur für „Neufälle“ gilt, während für „Altfälle“ das bisherige Recht anzuwenden ist, so dass die nachträgliche Sicherungsverwahrung nicht nur im Jugendstrafrecht, sondern für Fälle vor dem 1.1.2011 weiterhin möglich bleibt. 
Die Bundesministerin für Justiz hält die Neuregelung in der Abgrenzung zwischen Freiheit und Sicherheit für „zielgenau“ und sieht in dem zivilrechtlichen Therapieunterbringungsgesetz einen dritten Weg zur stationären Unterbringung ohne Strafcharakter für psychisch gestörte Personen. Für den Freistaat Bayern hob die Staatsministerin die Verbesserung des Abstandsgebots zwischen Straf- und Maßregelvollzug und die Besserstellung von Sicherungsverwahrten in der JVA Straubing - übertrieben - positiv hervor. Auffällig war, dass die Politikvertreterinnen und -Vertreter auf konkrete Fragen des Gerichts zum Abstandsgebot, zu Einzelheiten der Therapierung und Unterbringung psychisch gestörter Gewalttäter nach dem ThUG sowie zur Beibehaltung der JGG-Regelungen zur Sicherungsverwahrung nur wenig präzise und kaum überzeugend antworten konnten.

Als Sachverständige wurden Henning Radtke zur Zweispurigkeit von Strafen und Maßregeln der Besserung und Sicherung, für deren Beibehaltung er nachdrücklich eintrat, und Axel Dessecker zur Empirie der Sicherungsverwahrung gehört $(1990=182,2000=219$, $2010=536$ Sicherungsverwahrte, davon nur etwa $15 \%$ in Sozialtherapie). Auf die Frage einer deliktszentrierten Therapie ging auch Volker Dittmann ein, der die Rückfallquote von gegenwärtig 15 - 20 $\%$ bei Sexualtaten für halbierbar hält, wenn man anstelle unsicherer bzw. falscher Prognosen (3 von 4 = falsch) eine evidenzbasierte Risikoanalyse vornimmt, um Risikosituationen einschätzen zu können („Wir formulieren ein Risiko, ob es sich realisiert, ist eine andere Frage“). Für die Niederlande berichtete Pieter Tak über die Möglichkeit der „Überlassung mit behördlicher Unterbringung“, über forensisch-psychiatrische Zentren (FPZ) und über „long-stay-Einrichtungen" für nicht therapierbare Straftäter. Die Praxisprobleme im Vollzug der Sicherungsverwahrung wurden im Sachverständigen-Beitrag von Thomas Rösch (JVA Freiburg) besonders deutlich. Durch Verbesserung im Vollzug der Freiheitsstrafen hätten sich Strafe und Maßregel in den letzten Jahren eher angenähert, was zu dem Problem der Einhaltung des Abstandsgebots führt. Er plädiert für eine Personalausstattung bei 60 Sicherungsverwahrten mit drei Psychologen, vier Sozialarbeitern, einem Arbeitstherapeuten und 22 Beamten des allgemeinen Vollzugsdienstes, für eigene Gebäude mit einem eigenen Hof außerhalb der Ringmauern, für zehn Stunden Besuch pro Monat, für Aufhebung der Arbeitspflicht, für höheres Taschengeld, für die Einbeziehung in die Sozialversicherung und für ein überzeugendes Integrationsmanagement und eine gelingende, verbesserte Kooperation mit Nachsorgeeinrichtungen.

Was blieb nach einem langen, bis 18 Uhr dauernden Verhandlungstag mit intensiven und beeindruckenden Fragestellungen des Bundesverfassungsgerichts als Einschätzung? Die Erwartungen damals unterscheiden sich erheblich vom Ergebnis. Dachten die Beobachter damals, es bliebe bei Randkorrekturen, hat sich nun gezeigt, dass der Zweite Senat das gesamte Recht der Sicherungsverwahrung über den Haufen geworfen hat. Am 8.2.2011 schien es, als betreffe das Verdikt nur die jugendstrafrechtlichen Regelungen zur Sicherungsverwahrung. Man glaubte, das Therapieunterbringungsgesetz dürfte kaum eine größere praktische Bedeutung erlangen. Nun zeigt sich, dass das ThUG ein ernst zu nehmendes Modell ist. Wir sind gespannt, wie die Gesetzgebung reagieren wird.

NDR 1 Welle Nord, 28.12.2010

\section{Weitere Sexualstraftäter im Ameos-Klinikum?}

Ein unbefristeter Vertrag zwischen Schleswig-Holstein und dem Ameos-Klinikum in Neustadt (Kreis Ostholstein) sichert seit Montag die Betreuung von zwei Sexualstraftätern. Die beiden waren im August in Lübeck aus der nachträglichen Sicherungsverwahrung entlassen worden. Der neue Vertrag sieht mehr Polizei, geregelte Therapie und Kosten von etwa 250.000 Euro pro Jahr und Patient vor. Jetzt schließt das Ameos-Klinikum nicht aus, weitere Sexualstrafttäter aufzunehmen. „Wir denken, dass wir durchaus in der Lage sind, hier am Standort weitere Klienten zu betreuen“, sagte Geschäftsführer Michael Dieckmann der NDR 1 Welle Nord.

\section{Mehr als drei Millionen Euro bereitgestellt}

Nach einem Urteil des Europäischen Gerichtshofs für Menschenrechte (EGMR) sitzen in Schleswig-Holstein mindestens fünf weitere Gewaltverbrecher zu Unrecht hinter Gittern. Sie müssen in den kommenden Monaten entlassen werden. Schleswig-Holstein hat im nächsten Haushalt mehr als drei Millionen Euro für die Betreuung ehemaliger Sicherungsverwahrter bereitgestellt. Die Innenminister der norddeutschen Länder diskutieren zurzeit über einen gemeinsamen Standort. Dass dieser das Klinikum in Neustadt sein könnte, sieht Dieckmann bislang aber nicht.

Quelle: http://www.ndr.de/regional/schleswig-holstein/sicherungsverwahrung189.html 